\title{
Image beam from a wire laser
}

\author{
E. E. Orlova* \\ Institute for Physics of Microstructures, Russian Academy of Sciences, Nishny Novgorod GSP-105, 603950 Russia \\ and Lobachevskii State University, Nizhni Novgorod 603950, Russia
}

\author{
J. N. Hovenier, ${ }^{\dagger}$ P. J. de Visser, and J. R. Gao ${ }^{\ddagger}$ \\ Kavli Institute of NanoScience Delft, Faculty of Applied Sciences, Delft University of Technology, \\ Lorentzweg 1, 2628 CJ, Delft, The Netherlands
}

(Received 19 September 2014; published 12 May 2015)

\begin{abstract}
We demonstrate the formation of a narrow beam from a long $(L \gg \lambda)$ laser with subwavelength transverse dimensions (wire laser) as an image of the subwavelength laser waveguide formed by a spherical lens. The beam is linearly diverging with the angle determined by the ratio of the wavelength to the lens radius, while the minimum beam spot size is the same as that of the image of a point source. We realize such a beam experimentally using a terahertz quantum cascade wire laser.
\end{abstract}

DOI: 10.1103/PhysRevA.91.051802

PACS number(s): 42.60.-v, 42.25.Fx, 78.67.-n

A large group of lasers based on semiconductor nanowires [1], plasmon waveguides [2], and multi-quantum-well structures [3] have a wire geometry with transverse dimensions smaller than the emission wavelength and a length much larger than the wavelength. The spatial structure of wire laser radiation differs drastically from that of conventional lasers with large apertures. It was found to be highly divergent, with strong intensity modulations observed both in far [4] and near field [5], with the pattern of modulations more dense for longer lasers. It was shown that the far-field pattern of wire lasers is formed by the interference of radiation from the longitudinal distribution of sources along the laser waveguide [6] and is similar to that of antennas of traveling wave. Thus it depends strongly on the longitudinal phase velocity of the waveguide mode. Concentration of radiation into a narrow, axially symmetric beam along the laser axis with the divergence determined by the ratio of the wavelength to the laser length has been predicted by the antenna model for the modes of wire lasers with longitudinal phase velocity close to that of light in air, where all the sources along the laser emit in phase in the direction of the longitudinal axis. However, realization of such modes is hindered due to their low confinement and high radiation losses. Directive emission from wire lasers has been achieved using gratings with appropriate periodicity, acting as a discrete array of phased sources along the laser waveguide [7-10]. However, this approach not only implies a complicated waveguide design, but also leads to an increase of radiation losses; thus it is not always possible. Transformation of radiation with external optical elements is free from these complications. Beam shaping is a wellestablished technique for lasers with transverse dimensions much bigger than the wavelength [11]. Lenses have been used to collimate the radiation from wire lasers [12] and to provide their optical images [5,13], yet little is known about peculiarities of external transformation of wire laser radiation.

\footnotetext{
*orlova@ipm.sci-nnov.ru

${ }^{\dagger}$ Deceased.

${ }^{\ddagger}$ Also with SRON National Institute for Space Research, Utrecht, The Netherlands.
}

In this Rapid Communication we analyze the structure of the beam formed as an image of a wire laser placed on the axis of a spherical lens behind the focal plane (Fig. 1). According to geometrical theory of optical images, the image of the laser is stretched along the lens axis. The length of the image increases with reduction of the distance between the lens and the laser, and the image becomes semi-infinite when the end of the laser touches the focal plane. The distribution of the radiation field in the vicinity of the image of a wire laser is analyzed below using the methods of scalar diffraction theory. We find that the image of a wire laser can form a narrow beam with peculiar diffraction properties: it diverges linearly with an angle determined by the ratio of the wavelength to the lens radius, while the minimum beam spot size is close to that of the image of a point source that is much smaller than the radius of the lens. We realize such a beam experimentally using a terahertz quantum cascade laser.

Calculation of the image of a wire laser cannot be performed with the standard aperture diffraction methods [14], which have been used to develop the beam shaping techniques for the lasers with the apertures much larger than the wavelength [11], since a considerable part of radiation of wire lasers propagates outside the laser waveguide. This part of the radiation carries information about the longitudinal structure of the laser mode and enables the formation of the linear image. The influence of the longitudinal structure of the waveguide on the radiation distribution can be adequately described using the approach based on the equivalence of displacement currents in dielectrics and conductivity currents. It can be shown [15] that the radiation field in a dielectric medium is the same as the field in vacuum produced by equivalent current sources with the complex amplitudes (Gaussian units): $\vec{J}_{e q}=\vec{J}_{c}+j \omega(\varepsilon-1) \vec{E} / 4 \pi$, where $\vec{J}_{c}$ is the conduction current distribution, $\vec{E}$ the electric field, $\varepsilon$ the dielectric constant (we assume the time dependence of the field $e^{j \omega t}$ ). Thus the radiation field of a wire laser can be expressed in terms of the field values within the laser waveguide. Like in [6], we assume that each Cartesian component of equivalent current density, corresponding to an optical mode of a wire laser, can be presented by a combination of traveling waves along the laser axis:

$$
J_{e q}^{\kappa}\left(\vec{r}_{s}\right)=J_{\perp}^{\kappa}\left(\vec{\rho}_{s}\right)\left[\exp \left(j q z_{s}\right) \pm \exp \left(-j q z_{s}\right)\right],
$$




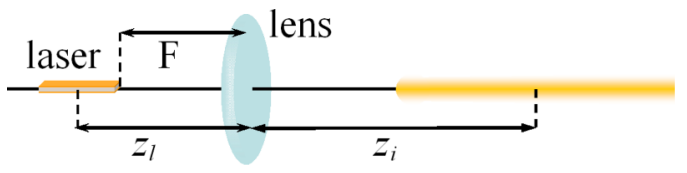

FIG. 1. (Color online) The scheme of the optical system.

where $\vec{\rho}_{s}$ and $z_{s}$ are the transverse and the longitudinal components of the radius vector $\vec{r}_{s}$ from the center of the laser to a point inside the laser cavity, $\left|z_{s}\right| \leqslant L / 2, L$ is the laser length, and $q$ is the wave number of the mode.

Distribution of the field produced by each traveling wave component of the equivalent current source (1) within the aperture of a lens placed in the far field of the laser at a distance much larger than the lens radius is given by

$E^{\kappa}=-\frac{j k L}{c} \frac{\exp \left(-j k r_{l}\right)}{z_{l}} \frac{\sin \left(\phi_{ \pm q} / 2-\Lambda \pi \rho_{l}^{2} / R^{2}\right)}{\phi_{ \pm q} / 2-\Lambda \pi \rho_{l}^{2} / R^{2}} F_{\perp}^{\kappa}$,

with $\kappa=x, y ; k=\omega / c ; c$ the velocity of light in air; $R$ the lens radius; $\vec{r}_{l}$ the radius vector from the reference point inside the laser cavity to the point in the lens plane; $z_{l}$ and $\rho_{l}$ the longitudinal and the transverse components of $\vec{r}_{l}$; and $F_{\perp}^{\kappa}=\int \vec{J}^{\kappa} \perp\left(\vec{\rho}_{s}\right) \exp \left(j \vec{k}_{\perp} \vec{\rho}_{s}\right) d \vec{\rho}_{s}$, with $\vec{k}=k \vec{r}_{l} / r_{l}$ the factor accounting for the influence of the transverse distribution of equivalent current on the radiation field structure. Due to the subwavelength transverse size of the waveguide, $F_{\perp}^{\kappa}$ varies slowly in the lens plane, and for a nonzero integral over the transverse distribution of equivalent current $I_{\perp}^{\kappa}=$ $\int \boldsymbol{J}_{\perp}^{\kappa}\left(\vec{\rho}_{s}\right) d \vec{\rho}_{s}$, assumed hereafter, the transverse factor can be approximated by $F_{\perp}^{\kappa} \simeq I_{\perp}^{\kappa}$. The two dimensionless parameters used in Eq. (2) are

$$
\phi_{ \pm q}=(k \pm q) L
$$

the phase shift between the traveling wave component of the waveguide mode and radiation in air propagating along the laser, and

$$
\Lambda=L / \Delta z_{l},
$$

the laser length normalized to the lens axial resolution, defined as the distance between the two point sources for which the maximum of one point spread function coincides with the first minimum of the second point spread function, $\Delta z_{l}=$ $2 \lambda z_{l}^{2} / R^{2}$. As we show below, (3) and (4) are the two parameters that determine the structure of the image of a wire laser.

Distribution of the radiation field produced by the components of the equivalent current (1) after transformation by the lens at the distances from the lens plane $z_{i}$ much bigger than the wavelength and small propagation angles can be obtained using the Kirchhoff integral over the aperture of the lens:

$$
E^{\kappa}\left(\vec{r}_{i}\right)=\frac{1}{j \lambda} \int E_{l}^{\kappa}\left(\rho_{l}\right) \frac{\exp \left(-j k\left|\vec{r}_{i}-\vec{r}_{l}\right|\right)}{\left|\vec{r}_{i}-\vec{r}_{l}\right|} d \vec{\rho}_{l},
$$

where $\vec{r}_{i}$ is the radius vector from the reference point inside the laser cavity to the point of observation in the image space, $E_{l}^{\kappa}\left(\rho_{l}\right)=E^{\kappa}\left(\rho_{l}\right) \exp \left(j k \rho_{l}^{2} / 2 F\right) G\left(\rho_{l}\right), F$ is the focal distance of the lens, and $G\left(\rho_{l}\right)$ is a pupil function, where $G=0$ beyond the lens radius $R$, and $G=1$ for $\rho_{l} \leqslant R$.

An expression for the image field distribution obtained by substitution of (2) into (5) can be simplified using the following considerations. First, a paraxial approximation can be used in the vicinity of the image of a wire source stretched along the lens axis: $\rho_{i} \ll z_{i}$ and $R \ll z_{i}$, where $\rho_{i}$ is the length of the radial component of $\vec{r}_{i}$. It allows us to use the expansion of $\left|\vec{r}_{i}-\vec{r}_{l}\right|$ as a power series of $\left|\vec{\rho}_{i}-\vec{\rho}_{l}\right| / z_{i}$, leaving the first two terms of this expansion. Additionally, as we are dealing with the far-field image, we can assume that in the vicinity of the image $\left|z_{c}-z_{l}\right| \ll z_{l}$, where $z_{c}=z_{i} F /\left(z_{i}-F\right)$ is the distance from the lens plane to the point conjugate to the observation point. The field amplitude in the vicinity of the image produced by $+q$ and $-q$ traveling wave components of the laser mode is then given by

$$
E^{\kappa}\left(\vec{r}_{i}\right)=C^{\kappa}\left(\vec{r}_{i}\right) \Phi_{ \pm q}\left(\vec{r}_{i}\right),
$$

where the first factor $C^{\kappa}\left(\vec{r}_{i}\right)$ is the paraxial approximation of a spherical wave from the center of the lens,

$$
C^{\kappa}\left(\vec{r}_{i}\right)=A^{\kappa} \exp \left(-j k z_{i}-k \rho_{i}^{2} / 2 z_{i}\right) / z_{i},
$$

with the amplitude $A^{\kappa}=-2 \pi \Lambda I_{\perp}^{\kappa} z_{l} \exp \left(-j k z_{l}\right) k / c$. The structure of the image is determined by the factor

$$
\Phi_{ \pm q}\left(\vec{r}_{i}\right)=\int_{0}^{1} J_{0}(\rho \sqrt{x}) e^{j 2 \pi z x} \frac{\sin \left(\phi_{ \pm q} / 2-\Lambda \pi x\right)}{\phi_{ \pm q} / 2-\Lambda \pi x} d x .
$$

Here $\rho$ and $z$ are the radial and the axial dimensionless coordinates in the image space defined as

$$
\rho=k R \rho_{i} / z_{i}, \quad z=\left(z_{c}-z_{l}\right) / \Delta z_{l} .
$$

The position of the image within the geometrical optics approximation corresponds to $|z| \leqslant \Lambda / 2$.

Let us now analyze the structure of the image beam. We consider below the $-q$ component of the equivalent current, propagating in the direction towards the lens, as it always produces the bigger impact to the image field. First we note that the absolute value of the field amplitude (6) at any $z_{i}$ depends only on the combination $k R \rho_{i} / z_{i}$. This means that in the case of the uniform structural factor (8) as a function of $z$, with the width of the peak of transverse distribution $\Delta \rho$, the image diverges linearly with $z_{i}$, with the width

$$
\Delta \rho_{i}=\frac{\Delta \rho}{2 \pi} \frac{\lambda}{R} z_{i} .
$$

However, the longitudinal structure of the image is not always uniform [see Figs. 2(a)-2(d)].

The longitudinal dependence of the structural factor (8) for slow modes with $\left|\phi_{-q}\right| \gg 2 \pi \Lambda$, which are typical for wire lasers, can be approximated by

$$
\left.\Phi_{-q}\right|_{\rho=0}=e^{-j \phi_{-q} / 2} \Phi_{p}(z+\Lambda / 2)-e^{j \phi_{-q} / 2} \Phi_{p}(z-\Lambda / 2),
$$

where

$$
\Phi_{p}(z)=\frac{j e^{j \pi z}}{\phi_{-q}} \frac{\sin (\pi z)}{\pi z}
$$

is proportional to the image field of a point source. The width of the central maximum of $\left|\Phi_{p}(z)\right|^{2}$ at the level of half maximum is $\Delta z^{p} \simeq 0.886$, and the distance between the maxima of (11) is equal to the normalized length of the laser $\Lambda$. For long lasers with the length larger than the double axial resolution of the lens $\Lambda>2$ [Fig. 2(a)], the maxima do not overlap, and the field amplitude in the center of the image is about $4 / \pi \Lambda$ smaller 


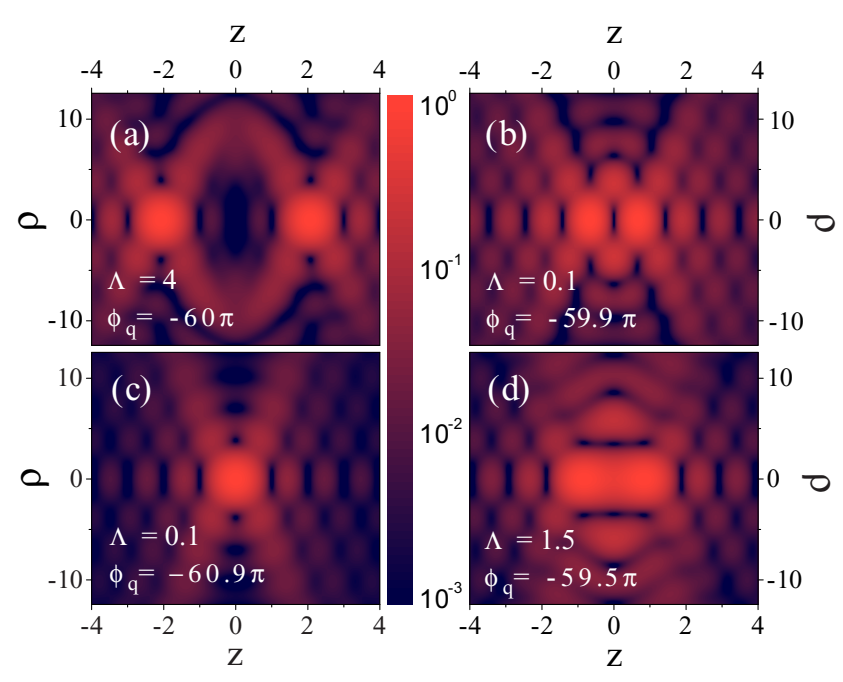

FIG. 2. (Color online) Square modulus of the structural factor for the main types of the wire laser image structure: two point sources (a), dipole (b), point source (c), and linear (d); $\rho$ and $z$ are the normalized transverse and axial coordinates.

than that of the maxima at the edges. For shorter lasers, or, equivalently, for the lenses with a larger axial resolution, the maxima overlap. However, even when $\Lambda \ll 2$, zero amplitude in the center of the image is possible ["dipole" image on Fig. 2(b)] when $\pi \Lambda-\phi_{-q}=2 n \pi$ with integer $n$, because of destructive interference of the two components in (11). Otherwise, the image for the laser with $\Lambda \ll 2$ is similar to that of a point source [Fig. 2(c)]. Overlapping maxima of (11) form an extended linear image with the normalized length

$$
\Delta z^{l} \simeq \Lambda+\Delta z^{p}
$$

at the level of half maximum of the square modulus of the structural factor when $\pi \Lambda-\phi_{-q} \simeq(2 n+1) \pi$ and $\Delta z^{p}<\Lambda \leqslant 1.5$ [Fig. 2(d)].

The radial dependence of the structural factor of the extended image does not change much within the image length (13) and is close to the radial dependence in the center of a point source image,

$$
\left.\Phi_{-q}\right|_{z=0}=\frac{2 L \sin \left(\phi_{-q} / 2\right)}{z_{l} \phi_{-q}} \frac{J_{1}(\rho)}{\rho},
$$

with the half width at half maximum of the square modulus of the structural factor,

$$
\Delta \rho \simeq 1.616 .
$$

Thus, according to (10), a linearly diverging wire image beam is formed within the interval of $z_{i}$, corresponding to (13) with the divergence

$$
\alpha \simeq 0.257 \lambda / R,
$$

while the minimum radius of the beam spot is equal to the width of a point source image located near the beam waist,

$$
a \simeq 0.257 \lambda z_{w} / R,
$$

where $z_{w}$ is the distance from the lens to the start of the image. The diffraction length of a wire laser image beam determined as a distance over which the beam increases its cross-sectional area by a factor of 2 is

$$
L_{d} \simeq 1.611 \frac{a R}{\lambda},
$$

provided the length of the image is larger.

Since the radius of the beam waist of the wire laser image beam (17) can be made much smaller than the lens radius, the divergence of such a beam determined by the ratio of the wavelength to the lens radius (16) is much smaller than the divergence of a Gaussian beam $\alpha_{G}$ with the same size of the beam waist, $\alpha / \alpha_{G} \simeq 0.8 a / R$, and the diffraction length of the wire laser image beam is much larger than Rayleigh range $Z_{R}, L_{d} / Z_{R} \simeq 0.5 R / a$.

The remarkable stability of the narrow beam width of the wire laser image beam resembles that of the Bessel beam. The dependence of the diffraction length of the wire laser image beam (18) on the radius of the lens aperture and the beam spot radius is similar to that of the propagation distance of truncated Bessel beams [16]. This reflects a similar mechanism of formation of the narrow field intensity maximum as a result of the interference of radiation propagating under an angle to the axis. It should be underlined that while truncated Bessel beams diverge fast beyond the final propagation distance, the propagation range of the image beam from a wire laser placed just behind the lens focal plane is infinite.

Let us compare an infinite wire laser image beam to a beam from a point source located in the focus of the lens [Figs. 3(a) and 3(b)]. The far-field divergence determined by the ratio of the wavelength to the lens radius is the same for both beams. The ratio of the minimum beam spot radius of a wire laser image beam (17) to the minimum spot radius of the beam from a point source which is equal to the lens radius can be expressed in terms of the Fresnel number of the beam

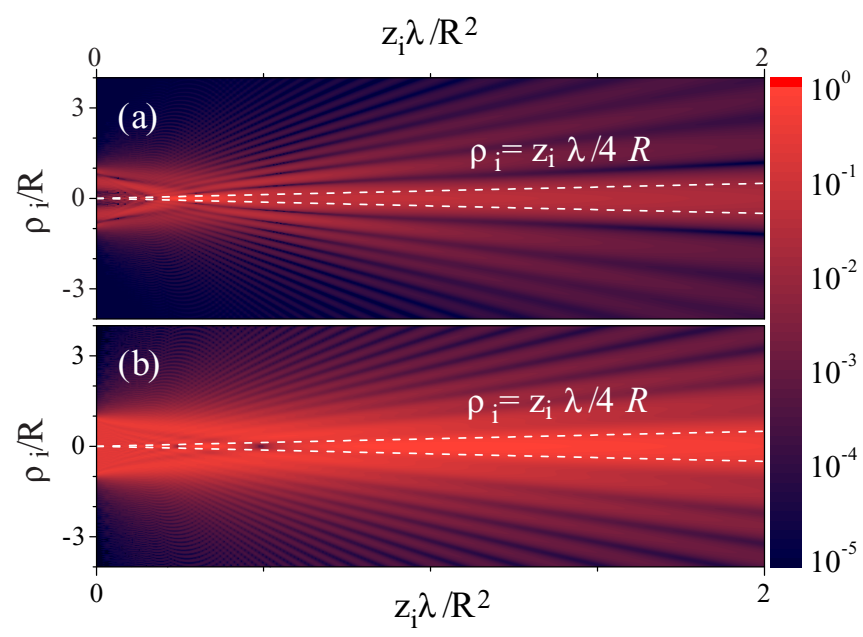

FIG. 3. (Color online) Numerically calculated field intensity distribution after transformation by a spherical lens with the radius $R=150 \lambda$ and focal distance $F=300 \lambda$, (a) for a wire laser of the length $L=15 \lambda$ with the laser mode wave vector $q=3.4 k$ placed with the end in the lens focal point, and (b) for a point source in the focal point of the lens. $z_{i}$ and $\rho_{i}$ are the longitudinal and the transverse components of the radius vector from the center of the lens to the observation point. 


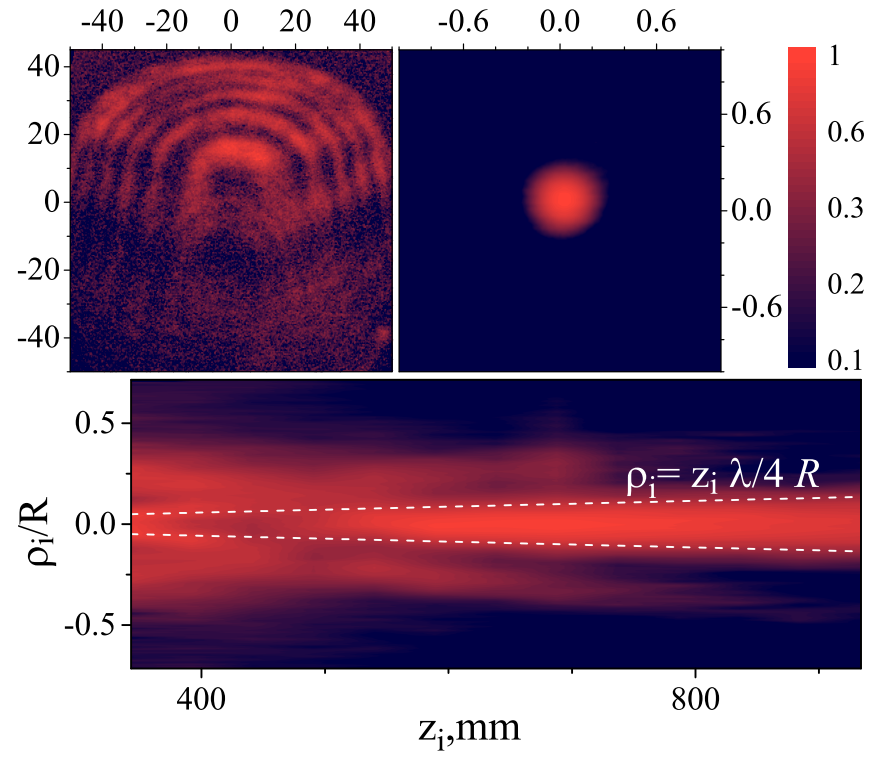

FIG. 4. (Color online) Normalized far-field intensity of the terahertz wire laser measured with an angular resolution of $2.6 \mathrm{deg}$ (left), far-field radiation intensity of the same laser placed with one end in focus of a spherical lens with the radius $R=13.75 \mathrm{~mm}$ measured with an angular resolution of $0.086 \mathrm{deg}$ (right). An equirectangular projection has been used; scales are given in degrees. Normalized field intensity distribution in the plane containing the lens axis measured with $3 \mathrm{~mm}$ transverse resolution. $z_{i}$ and $\rho_{i}$ are the longitudinal and the transverse components of the radius vector from the center of the lens to the observation point (bottom).

waist: $a / R \simeq 1 / 4 N_{F}\left(z_{w}\right)$. The maximum value of the Fresnel number of the beam waist of an infinite wire laser image beam can be obtained using (13) for the length of the image with the condition of the uniform longitudinal structure of the beam $\Lambda \leqslant 1.5: N_{F}\left(z_{w}\right) \simeq 4.8$. Thus the minimum beam spot radius of a wire laser image beam is about 20 times smaller than that of a beam formed from a point source, and the minimum divergence of an infinite wire laser image beam is about 20 times smaller than the ratio of the wavelength to the minimum beam spot radius.

We realize a wire laser image beam experimentally using a single-mode terahertz $(\lambda=109.3 \mu \mathrm{m})$ quantum cascade laser with a subwavelength aperture of $10 \times 25 \mu \mathrm{m}$ and length $L=1.5 \mathrm{~mm}$, based on a standard "metal-metal" waveguide design [17]) with uncoated front, back, and side facets. The farfield pattern of the laser measured with a setup similar to [4] is presented in Fig. 4(a). Strong modulations of far-field intensity are almost axially symmetrical, with the separation of intensity rings in agreement with the wire laser model [6]. Radiation of the laser was partially blocked by the sample holder, leading to a smaller intensity in the lower part of Fig. 4(a). A narrow beam was obtained when this laser was placed along the axis of a spherical lens with a focal distance $F=26.5 \mathrm{~mm}$ and a radius $R=13.75 \mathrm{~mm}$ with the end facet near the focus. The far-field intensity of this beam, measured in the focal plane of an additional spherical lens, is presented in Fig. 4(b). The angular width of this beam of $0.16 \mathrm{deg}$ at half maximum was measured using the detector providing an angular resolution of $0.086 \mathrm{deg}$. The angular resolution was limited by the signalto-noise ratio of the detector. The measured radius of the beam waist $\left(z_{w} \simeq 595 \mathrm{~cm}\right)$ was about $2.2 \mathrm{~mm}$ and the beam was diverging linearly in agreement with the model [Fig. 4(c)]. The difference between the measured and the theoretical values of the beam width is within $50 \%$, and a large part of this difference can be attributed to the measurement resolution due to the finite aperture size of the detector.

In conclusion, we demonstrated that a narrow beam can be obtained as an image of a wire laser formed with a spherical lens. The wire laser image beam combines small divergence, which is determined by the ratio of the wavelength to the radius of the lens, with a small beam spot radius, coinciding with the width of the image of a point source. Such beams can be useful for applications where high transverse resolution is needed for a large range of distances. The wire laser image beam may also provide new opportunities for optical manipulation, as an array of such beams with separately tuned dynamical parameters can be obtained as an image of an array of wire lasers.

We acknowledge the use of quantum cascade lasers provided by Prof. Qing Hu at Massachusetts Institute of Technology, USA, and constructive and helpful discussions with T. M. Klapwijk, M. I. Petelin, and V. I. Talanov. E.E.O. gratefully acknowledges an NWO visiting grant and an Erasmus Mundus grant. This research is supported by NATO's Public Diplomacy Division in the frame of "Science for Piece" Project No. 984068, Swiss National Science Foundation Project No. IZ73Z0 152761, NWO-RFBR Centre of Excellence for the Detection of Terahertz Radiation (TeraDec), and RFBR Project No. 14-02-00979a.
[1] R. Yan, D. Gargas, and P. Yang, Nanowire photonics, Nat. Photon. 3, 569 (2009).

[2] R. F. Oulton, V. J. Sorger, T. Zentgraf, R.-M. Ma, C. Gladden, L. Dai, G. Bartal, and X. Zhang, Plasmon lasers at deep subwavelength scale, Nature (London) 461, 629 (2009).

[3] B. S. Williams, Terahertz quantum-cascade lasers, Nat. Photon. 1, 517 (2007).

[4] J. L. Adam, I. Kasalynas, J. N. Hovenier, T. O. Klaassen, J. R. Gao, E. E. Orlova, B. S. Williams, S. Kumar, Q. Hu, and J. L. Reno, Beam pattern of terahertz quantum cascade lasers with sub-wavelength cavity dimensions, Appl. Phys. Lett. 88, 151105 (2006).
[5] L. K. van Vugt, S. Rühle, and D. Vanmaekelbergh, Phasecorrelated nondirectional laser emission from the end facets of a ZnO nanowire, Nano Lett. 6, 2708 (2006).

[6] E. E. Orlova, J. N. Hovenier, T. O. Klaassen, I. Kasalynas, A. J. L. Adam, J. R. Gao, T. M. Klapwijk, B. S. Williams, S. Kumar, Q. Hu, and J. L. Reno, Antenna model for wire lasers, Phys. Rev. Lett. 96, 173904 (2006).

[7] M. I. Amanti, M. Fischer, G. Scalari, M. Beck, and J. Faist, Low-divergence single-mode terahertz quantum cascade laser, Nat. Photon. 3, 586 (2010).

[8] L. Mahler, A. Tredicucci, F. Beltram, C. Walther, J. Faist, H. E. Beere, D. A. Ritchie, and D. S. Wiersma, Quasi- 
periodic distributed feedback laser, Nat. Photon. 4, 165 (2010).

[9] T.-Y. Kao, Q. Hu, and J. L. Reno, Perfectly phase-matched third-order DFB THz quantum-cascade lasers, Opt. Lett. 37, 2070 (2012).

[10] M. Cui, J. N. Hovenier, Y. Ren, N. Vercruyssen, J. R. Gao, T. Y. Kao, Q. Hu, and J. L. Reno, Beam and phase distributions of a terahertz quantum cascade wire laser, Appl. Phys. Lett. 102, 111113 (2013).

[11] Laser Beam Shaping Theory and Techniques, edited by F. H. Dickey and S. C. Holswade (Marcel Dekker, Inc., New York, 2000).

[12] A. W. M. Lee, Q. Qin, S. Kumar, B. S. Williams, Q. Hu, and J. L. Reno, High-power and high-temperature THz quantum-cascade lasers based on lens-coupled metal-metal waveguides, Opt. Lett. 32, 2840 (2007).
[13] D. J. Sirbuly, M. Law, H. Yan, and P. Yang, Semiconductor nanowires for subwavelength photonics integration, J. Phys. Chem. B 109, 15190 (2005).

[14] M. Born and E. Wolf, Principles of Optics: Electromagnetic Theory of Propagation, Interference and Diffraction of Light, 9th ed. (Pergamon, Oxford, 1999).

[15] G. A. Thiele, in Computer Techniques for Electromagnetics, edited by R. Mitra (Pergamon, Elmsford, NY, 1973), Chap. 2.

[16] J. Durnin, J. J. Miceli, and J. H. Eberly, Diffraction-free beams, Phys. Rev. Lett. 58, 1499 (1987).

[17] B. S. Williams, S. Kumar, H. Callebaut, Q. Hu, and J. L. Reno, Terahertz quantum-cascade laser at $100 \mu \mathrm{m}$ using metal waveguide for mode confinement, Appl. Phys. Lett. 83, 2124 (2003). 